\title{
Reeder's Conjecture for Even Orthogonal Lie Algebras
}

\author{
Sabino Di Trani ${ }^{1}$ \\ Received: 16 August 2021 / Accepted: 7 January 2022 \\ (C) The Author(s) 2022
}

\begin{abstract}
In the paper we complete a case by case proof of Reeder's Conjecture started in our previous work, proving the conjecture for simple Lie algebras of type $D$ and for the exceptional cases.
\end{abstract}

Keywords Simple lie algebras · Exterior algebra $\cdot$ Small representations ·

Graded multiplicities $\cdot$ Zero weight space representations

Mathematics Subject Classification (2010) 17B10 $17 \mathrm{~B} 20 \cdot 17 \mathrm{~B} 22$

\section{Introduction}

The structure of $\mathfrak{g}$ - representation of the exterior algebra $\Lambda \mathfrak{g}, \mathfrak{g}$ a simple Lie algebra over $\mathbb{C}$, has been extensively studied in the last fifty years (see [14, 17] and [18] for many relevant results on this topic). Despite its finite dimensionality, an uniform description of irreducible components of $\Lambda \mathfrak{g}$ is known only in a conjectural form.

In '97 Reeder proved that for the irreducible representations $V_{\lambda}$ indexed by certain dominant weights $\lambda$ called small (i.e., such that $\lambda$ is in the root lattice and $2 \alpha$ is not smaller than $\lambda$ in the dominant order for all positive roots $\alpha$ ), the multiplicity of $V_{\lambda}$ in $\Lambda \mathfrak{g}$ equals $2^{\text {rkg }} m_{\lambda}^{0}$, where $m_{\lambda}^{0}$ is the dimension of the zero weight space in $V_{\lambda}$. Moreover, inspired by the work of Broer about small representations in the symmetric algebra (see [5]), he conjectured that the problem of determining the graded multiplicities of small representations in the exterior algebra can be reduced to a problem involving Weyl group representations on the zero weight space $V_{\lambda}^{0}$.

The setup for the conjecture is the following: let $\mathfrak{g}$ be a simple Lie algebra over $\mathbb{C}$, fix a Cartan subalgebra $\mathfrak{h}$ and let $\Phi$ be the associated root system, with Weyl group $W$. We denote by (_, , ) the $W$-invariant positive-definite inner product on $\mathfrak{h}^{*}$ induced by the Killing form and by $\alpha^{\vee}$ the coroot associated to $\alpha$. We choose a set of positive roots $\Phi^{+}$associated to a simple system $\Delta$. Let $\rho$ be the corresponding Weyl vector and $\theta$ (resp. $\theta_{s}$ ) the highest root (resp. the highest short root). We will denote by $\Pi^{+}$the set of dominant weights, $\omega_{i}$ will

Presented by: Peter Littelmann

The author has been partially supported by GNSAGA - INDAM group.

Sabino Di Trani

sabino.ditrani@uniroma1.it

1 Dipartimento di Matematica "Guido Castelnuovo", Sapienza - Università di Roma, Rome, Italy 
be the $i$-th fundamental weight. Let $H$ (resp $H^{h}$ ) be the space of $W$-harmonic polynomials (resp. of degree $h$ ) on $\mathfrak{h}$, i.e. polynomials annihilated by constant coefficients $W$-invariant differential operators with positive degree.

\section{Conjecture 1.1 (Reeder) Consider the two polynomials}

$$
\begin{gathered}
P\left(V_{\lambda}, \bigwedge \mathfrak{g}, u\right)=\sum_{n \geq 0} \operatorname{dim} \operatorname{Hom}_{\mathfrak{g}}\left(V_{\lambda}, \bigwedge^{n} \mathfrak{g}\right) u^{n}, \\
P_{W}\left(V_{\lambda}^{0}, q, y\right)=\sum_{n \geq 0} \operatorname{dim} \operatorname{Hom}_{W}\left(V_{\lambda}^{0}, \bigwedge^{k} \mathfrak{h} \otimes H^{h}\right) q^{h} y^{k} .
\end{gathered}
$$

If $V_{\lambda}$ is a small representation, then the following equality holds:

$$
P\left(V_{\lambda}, \bigwedge \mathfrak{g}, q\right)=P_{W}\left(V_{\lambda}^{0}, q^{2}, q\right)
$$

This conjecture was implicitly proved in type $A_{n}$ comparing the "First Layer Formulae" of Stembridge [21] with the results due to Kirillov, Pak [13] and Molchanov [16]. Moreover some general formulae for graded multiplicities are proved when $V_{\lambda}$ is the adjoint or the little adjoint module in [2, 7-9] and [22]. In these cases the description of the zero weight space is quite simple and the proof of the Reeder's Conjecture is completely straightforward using the formulae proved in [12]. In our previous paper [10] we proved the conjecture for Lie algebras of type $B$ and $C$ using the tools introduced by Stembridge in [22]. The aim of this note is to complete a case by case proof of the Reeder's Conjecture for the classical Lie algebras showing that the conjecture holds for even orthogonal Lie algebras. Furthermore, we checked using SageMath that the conjecture is true in the exceptional cases.

The first sections of the paper are dedicated to explain our tools and to make more explicit the "Weyl group part" of the conjecture. Section 4 is devoted to prove Conjecture 1.1 for even orthogonal Lie algebras. We use a mixed strategy with respect to what we have done in our previous paper: we use the combinatorics of weights and the action of the Weyl group to find nice closed expressions for the coefficients of Stembridge's minuscule recurrences and again we conclude using an inductive reasoning. Section 5 contains some technical results about a suitable reduction of the Stembridge's recurrences. Finally, in Section 6 we give an overview about small representations in the exceptional cases and describe the computational approach that we followed to check the conjecture.

\section{Stembridge's Recurrences}

Our work make an extensive use of results exposed in [22] about the the coefficients $C_{\mu}(q, t)$ in the character expansion of Macdonald kernels. The reason for our interest in these functions is that the evaluation $C_{\mu}\left(-q, q^{2}\right)$ gives the polynomial of graded multiplicities of $V_{\mu}$ in the exterior algebra.

Let $\Delta(q, t)$ denote the Macdonald kernel and define $C_{\mu}(q, t) \in \mathbb{C}\left[q^{ \pm 1}, t^{ \pm 1}\right], \mu \in \Pi^{+}$ by the relation $\Delta(q, t)=\sum_{\mu \in \Pi^{+}} C_{\mu}(q, t) \chi(\mu)$, where $\chi(\mu)$ denotes the irreducible character associated to the dominant weight $\mu$. It is possible to extend the Definition of $C_{\mu}(q, t)$ to any weight $\mu$ setting

$$
C_{\mu}(q, t)= \begin{cases}0 & \text { if } \mu+\rho \text { is not regular, } \\ (-1)^{l(\sigma)} C_{\lambda}(q, t) & \text { if } \sigma(\mu+\rho)=\lambda+\rho, \lambda \in \Pi^{+}, \sigma \in W .\end{cases}
$$


We will say that, if there exists $\sigma$ such that $\sigma(\mu+\rho)=\lambda+\rho$, the weight $\mu$ is conjugated to $\lambda$ and we will write $\mu+\rho \sim \lambda+\rho$. These rational functions $C_{\mu}(q, t)$ satisfy some recurrences. The problem of their explicit computation reduces to solving a linear system of equations with coefficients in $\mathbb{C}\left[q^{ \pm 1}, t^{ \pm 1}\right]$.

We recall that a weight (resp. coweight) $\omega$ is said to be minuscule if $\left(\omega, \alpha^{\vee}\right) \in\{0, \pm 1\}$ (resp. $(\omega, \alpha))$ for all positive roots. Fix a dominant weight $\lambda$. If $\omega$ is a minuscule coweight, then the following relation holds (see [22], formula (5.14)):

$$
\sum_{i=1}^{k} C_{w_{i} \lambda}(q, t)\left(\sum_{\psi \in O_{\omega}}\left(t^{-\left(\rho, w_{i} \psi\right)}-q^{(\lambda, \omega)} t^{\left(\rho, w_{i} \psi\right)}\right)\right)=0 .
$$

Here $w_{1}, \ldots, w_{k}$ are minimal coset representatives of $W / W_{\lambda}$, where $W_{\lambda}$ is the stabilizer of $\lambda$, and $O_{\omega}$ is the orbit $W_{\lambda} \cdot \omega$. We call this recursive relation the minuscule recurrence. The $C_{\mu}$ appearing in Eq. 2.2 are not necessary in their reduced form (i.e. the weight $\mu$ is not necessary dominant), but the reduced form can be always achieved according to the Definition 2.1. Considering only the reduced forms, Stembridge proves that the $C_{\mu}(q, t)$ appearing in Eq. 2.2 are indexed only by weights $\mu$ smaller or equal to $\lambda$ in the dominant order. We recall that if $\lambda$ is small, a weight $\mu \leq \lambda$ is again small. Consequently our strategy becomes clear: we determine closed formulae for the polynomials $P_{W}\left(V_{\lambda}^{0}, q, y\right)$ (explicitly computed in [12]), and then we prove by induction that these closed formulae satisfy Stembridge's recursive relations, specialized in $-q$ and $q^{2}$.

In [10] we used the minuscule recurrence to prove Reeder's Conjecture in type $B$. A different approach is needed to obtain a proof in type $C$, where Stembridge's quasi minuscule recurrence is more efficient. Our choice of using minuscule recurrence in type $D$ comes from a computational reason: in $D_{n}$ the fundamental weight $\omega_{1}$ is minuscule and it is consequently easier to reduce the polynomial coefficients to a simpler form.

\section{Small Representations and their Zero Weight Spaces}

We say that an irreducible representation $V_{\lambda}$ is small if its weight is small. We refer to [1] and [19] for a complete classification of small representations and their zero weights spaces as $W$ representations.

As in the case of hyperoctahedral group $B_{n}$ examined in [10], the irreducible representations of the Weyl group $D_{n}=S_{n} \ltimes(\mathbb{Z} / 2 \mathbb{Z})^{n-1}$ are encoded by pairs of partitions $(v, \mu), v \vdash k, \mu \vdash h, h+k=n$. In the $B_{n}$ case, the irreducible representations can be realized as $\pi_{v, \mu}=\operatorname{Ind}_{B_{k} \times B_{h}}^{B_{n}} \pi_{v}^{\prime} \times \pi_{\mu}^{\prime \prime}$, where, if $\pi_{\tau}$ is the irreducible $S_{p}$-module associated to $\tau \vdash p$, and $\varepsilon_{q}$ is the sign representation of $(Z / 2 \mathbb{Z})^{q}$, we have $\left(\pi_{v}^{\prime}\right)_{\mid S_{k}}=\pi_{v},\left(\pi_{v}^{\prime}\right)_{\mid(\mathbb{Z} / 2 \mathbb{Z})^{k}}=$ $1_{k},\left(\pi_{v}^{\prime \prime}\right)_{\mid S_{h}}=\pi_{\mu},\left(\pi_{\mu}^{\prime \prime}\right)_{\mid(\mathbb{Z} / 2 \mathbb{Z})^{h}}=\varepsilon_{h}$. All the irreducible representations of the Weyl group of type $D_{n}$ can be then obtained restricting to $S_{n} \ltimes(\mathbb{Z} / 2 \mathbb{Z})^{n-1}$ a representation of the form $\pi_{v, \mu}$. If $v \neq \mu$, the representation $\tilde{\pi}_{v, \mu}:=\operatorname{Res}_{D_{n}}^{B_{n}} \pi_{v, \mu}$ remains irreducible, otherwise $\operatorname{Res}_{D_{n}}^{B_{n}} \pi_{\nu, \nu}$ splits in two non isomorphic irreducible components that we denote by $\tilde{\pi}_{\nu, \mu}^{I}$ and $\tilde{\pi}_{v, v}^{I I}$. A complete description of zero weight spaces for small representations in type $D$ is displayed in Table $1 .^{1}$

\footnotetext{
${ }^{1}$ In Table 1 the irreducible representations are described by the associated pair of partitions $(\nu, \mu)$ when $v \neq \mu$. If $v=\mu$ we denote by $(\nu, v)_{I}$ and $(v, v)_{I I}$ the irreducible representations $\tilde{\pi}_{v, \mu}^{I}$ and $\tilde{\pi}_{v, v}^{I I}$, respectively.
} 
Table 1 Zero weights space of small representations: Type D

Small representation

Highest weight
Zero weight space

$(\nu, \mu)$ description

\begin{tabular}{ll}
\hline$\omega_{2 i}, i<\frac{n-1}{2}$ & $((n-i),(i))$ \\
$2 \omega_{n-1}, 2 \omega_{n} \quad(n$ even $)$ & $\left(\left(\frac{n}{2}\right),\left(\frac{n}{2}\right)\right)_{I I},\left(\left(\frac{n}{2}\right),\left(\frac{n}{2}\right)\right)_{I}$ \\
$\omega_{n-1}+\omega_{n} \quad(n$ odd $)$ & $\left(\left(\frac{n+1}{2}\right),\left(\frac{n-1}{2}\right)\right)$ \\
$2 \omega_{1}$ & $((n-1,1), \emptyset)$ \\
$\omega_{1}+\omega_{2 i+1}, i<\frac{n-1}{2}$ & $((n-i-1,1),(i)) \oplus((n-i-1),(i, 1))$ \\
$\omega_{1}+\omega_{n-1}+\omega_{n} \quad(n$ even $)$ & $\left(\left(\frac{n}{2}, 1\right),\left(\frac{n-2}{2}\right)\right) \oplus\left(\left(\frac{n}{2}\right),\left(\frac{n-2}{2}, 1\right)\right)$ \\
$\omega_{1}+2 \omega_{n-1}, \omega_{1}+2 \omega_{n-1} \quad(n$ odd $)$ & $\left(\left(\frac{n-1}{2}, 1\right),\left(\frac{n-1}{2}\right)\right)$ \\
\hline
\end{tabular}

Let us denote by $\bar{P}_{W}$ the polynomial $P_{W}\left(V_{\lambda}^{0}, q, y\right)$ divided by $\prod_{i=1}^{n}\left(1-q^{m_{i}+1}\right)$, where $m_{1}, \ldots, m_{n}$ are the exponents of Weyl group $W$. We will determine explicit expressions for the polynomial $\bar{P}_{W}$.

We will encode partitions $\lambda=\left(\lambda_{1}, \geq \lambda_{2}, \ldots, \geq, \lambda_{n}\right)$ by Young diagrams, displayed in the English way. Here $h(i j), c(i j)$ are the hook length and the content of the box $(i j)$ respectively; $|\lambda|$ and $n(\lambda)$ will denote the quantities $\sum_{i=1}^{n} \lambda_{i}$ and $\sum_{i=1}^{n}(i-1) \lambda_{i}$. We recall the following results about the $\bar{P}_{W}$ polynomials for the hyperoctahedral group:

Theorem 3.1 ([12], Proposition 3.3) Let $\pi_{\alpha, \beta}$ be the irreducible representation of the Weyl group $B_{n}$ indexed by the pair of partitions $(\alpha, \beta)$.

$$
\bar{P}_{B_{n}}\left(\pi_{\alpha, \beta} ; q, y\right)=q^{2 n(\alpha)+2 n(\beta)+|\beta|} \prod_{(i, j) \in \alpha} \frac{1+y q^{2 c(i j)+1}}{1-q^{2 h(i j)}} \prod_{(i, j) \in \beta} \frac{1+y q^{2 c(i j)-1}}{1-q^{2 h(i j)}} .
$$

We remark that the reflection representation of the hyperoctahedral group restrict to the reflection representation of the group $S_{n} \ltimes(\mathbb{Z} / 2 \mathbb{Z})^{n-1}$. Moreover, denoting by $\operatorname{Sgn}(n)$ the sign representation of $B_{n}$, the representation $\pi_{\alpha, \beta}$ and $\pi_{\beta, \alpha}=\pi_{\alpha, \beta} \otimes \operatorname{Sgn}(n)$ restrict to the same irreducible representation $\tilde{\pi}_{\alpha, \beta}$. As an immediate consequence, it is possible to obtain the following relations between the $P_{W}$ polynomials in type $B$ and in type $D$ :

$$
\begin{aligned}
& P_{D}\left(\tilde{\pi}_{\alpha, \beta} ; q, y\right)=P_{B}\left(\pi_{\alpha, \beta} ; q, y\right)+P_{B}\left(\pi_{\beta, \alpha} ; q, y\right) \\
& P_{D}\left(\tilde{\pi}_{\alpha, \alpha}^{I} ; q, y\right)=P_{D}\left(\tilde{\pi}_{\alpha, \alpha}^{I I} ; q, y\right)=P_{B}\left(\pi_{\alpha, \alpha} ; q, y\right)
\end{aligned}
$$

Now we want to rearrange the above formulae in a more useful way. Let $\lambda$ be a partition, we define

$$
S_{\lambda}(q)=\left(q^{n}+1\right) \prod_{(i j) \in \lambda}\left(1+y q^{2 c(i j)+1}\right) \quad R_{\lambda}(q)=\left(q^{n}+1\right) \prod_{(i j) \in \lambda}\left(q+y q^{2 c(i j)}\right)
$$

In the case of $\lambda=(k, 1)$ or $\lambda=(k)$, some nice relations hold:

$$
S_{(k, 1)}=S_{(k)} \frac{(q+y)}{q}, \quad R_{(k, 1)}=R_{(k)} \frac{\left(q^{3}+y\right)}{q^{2}}, \quad R_{(k)}=q^{k-1} S_{(k-1)}(q+y) .
$$


Now it is possible to express the $\bar{P}_{D}$ polynomials for zero weight space representations in Table 1 in a more compact way. Set $H(\lambda)=\prod_{(i j) \in \lambda}\left(1-q^{4 h(i j)}\right)$, where $(i j)$ ranges between the boxes of $\lambda$.

$$
\begin{aligned}
& \bar{P}_{D}\left(\tilde{\pi}_{(n-1,1), \emptyset} ; q, y\right)=\frac{q^{2}\left[R_{(n-1,1)}+S_{(n-1,1)}\right]}{H(n-1,1)}, \\
& \bar{P}_{D}\left(\tilde{\pi}_{(k),(n-k)} ; q, y\right)=\frac{S_{(k)} R_{(n-k)}+R_{(k)} S_{(n-k)}}{H(k) H(n-k)\left(1+q^{n}\right)} \\
& =\frac{q^{k-1} S_{(k-1)} S_{(n-k-1)}(q+y)\left(q^{n-2 k}+1\right)\left(1+y q^{n+1}\right)}{H(k) H(n-k)\left(1+q^{n}\right)}, \\
& \bar{P}_{D}\left(\tilde{\pi}_{(k, 1),(n-k-1)} \oplus \tilde{\pi}_{(n-k-1,1),(k)} ; q, y\right)=\frac{q^{2}\left[S_{(k)} R_{(n-k-1,1)}+R_{(k)} S_{(n-k-1,1)}\right]}{H(k) H(n-k-1,1)\left(1+q^{n}\right)} \\
& +\frac{q^{2}\left[S_{(n-k-1)} R_{(k, 1)}+R_{(n-k-1)} S_{(k, 1)}\right]}{H(n-k-1) H(k, 1)\left(1+q^{n}\right)} \\
& =\frac{q^{2} S_{(k-1)} S_{(n-k-2)}(q+y)}{H(k+1) H(n-k)\left(1+q^{n}\right)\left(1-q^{2}\right)} Q(n, k) \text {. }
\end{aligned}
$$

Here we set

$$
P(n, k, q, y)=q^{k-2}(q+y)\left(1+y q^{2(n-k-2)+1}\right)+q^{n-k-4}\left(q^{3}+y\right)\left(1+y q^{2(k-1)+1}\right)
$$

and

$$
\begin{aligned}
Q(n, k, q, y)= & P(n, k, q, y)\left(q^{2(k+1)}-1\right)\left(q^{2(n-k-1)}-1\right) \\
& +P(n, n-k-1, q, y)\left(q^{2 k}-1\right)\left(q^{2(n-k)}-1\right) .
\end{aligned}
$$

\section{Even Orthogonal Algebras}

We recall now the realization of root system $D_{n}$ as exposed in [4]. In an euclidean vector space with basis $\left\{e_{1}, \ldots, e_{n}\right\}$, consider the set $\Phi_{D_{n}}$ of vectors $\left\{ \pm e_{i} \pm e_{j}\right\}_{i \neq j, i, j \leq n}$. We fix a positive system of roots choosing the vectors $\left\{e_{i} \pm e_{j}\right\}_{i \neq j, i, j \leq n}$; according to such a description, the fundamental weights are $\omega_{i}=e_{1}+\cdots+e_{i}$ if $i<n-1, \omega_{n-1}=$ $\frac{1}{2}\left(e_{1}+\cdots-e_{n}\right)$ and $\omega_{n}=\frac{1}{2}\left(e_{1}+\cdots+e_{n}\right)$. Moreover the coweight $e_{1}$ is quasi minuscule and the Weyl vector is $\rho=\sum(n-j+1) e_{j}$. We have three different families of formulae for $P_{W}\left(V_{\lambda}^{0}, q^{2}, q\right)$, depending on the reducibility of representation $V_{\lambda}^{0}$. We will denote the polynomials $P_{D}\left(\tilde{\pi}_{(k),(n-k)} ; q^{2}, q\right)$ by $\mathbf{C}_{k, n}$, the polynomial $P_{D}\left(\tilde{\pi}_{(n-1,1), \emptyset} ; q^{2}, q\right)$ by $\mathbf{C}_{2 \mid 0, n}$ and the polynomials $P_{D}\left(\tilde{\pi}_{(k, 1),(n-k-1)} \oplus \tilde{\pi}_{(n-k-1,1),(k)} ; q^{2}, q\right)$ by $\mathbf{C}_{2 \mid k, n}$. Similarly we will denote by $C_{k, n}$ and $C_{2 \mid k, n}$ (or simply by $C_{k}$ and $C_{2 \mid k}$ when the context is clear) the rational functions appearing in the Stembridge's recurrences, associated respectively to weight of the form $\omega_{2 k}$ and $\omega_{1}+\omega_{2 k+1}$, and specialized in $-q$ and $q^{2}$. The following relations between the polynomials $\mathbf{C}_{k, n}$ and $\mathbf{C}_{2 \mid k, n}$ hold:

$$
\begin{gathered}
\mathbf{C}_{k+1, n}=\mathbf{C}_{k, n} \frac{q^{2}\left(1+q^{2(n-2 k-2)}\right)\left(1+q^{4 k-1}\right)\left(1-q^{4 n-4 k}\right)}{\left(1+q^{2 n-4 k}\right)\left(1+q^{4 n-4 k-5}\right)\left(1-q^{4 k+4}\right)}, \\
\mathbf{C}_{2 \mid k, n}=\mathbf{C}_{k, n} \frac{Q\left(n, k, q^{2}, q\right)}{q^{2 k-6}\left(1-q^{4}\right)\left(1-q^{4 k+4}\right)\left(1+q^{2 n-1}\right)\left(1+q^{2 n-4 k}\right)\left(1+q^{4 n-4 k-5}\right)}
\end{gathered}
$$


Remark 4.1 Observe that if we set $n=2 k$ in Eq. 4.1, the ratio $T_{k}^{n}$ between $\mathbf{C}_{k, n}$ and $\mathbf{C}_{k-1, n}$ becomes equal to

$$
\frac{2 \cdot q^{2}\left(1+q^{4 k-5}\right)\left(1-q^{4 k+4}\right)}{\left(1+q^{4}\right)\left(1+q^{4 k-1}\right)\left(1-q^{4 k}\right)}
$$

This is coherent with the fact that, if $n$ is even, the specialization of $P_{D}\left(\tilde{\pi}_{(k),(n-k)} ; q^{2}, q\right)$ in $k=\frac{n}{2}$ is equal to $2 P_{D}\left(\tilde{\pi}_{\left(\frac{n}{2}\right),\left(\frac{n}{2}\right)}^{I} ; q^{2}, q\right)$.

\subsection{Weights of the Form $\omega_{2 k}, 2 \omega_{n-1}, 2 \omega_{n}$ if $n$ is Even and $\omega_{n-1}+\omega_{n}$ if $n$ is Odd}

We consider the recurrence (2.2) and make the evaluations $q \rightarrow-q$ and $t \rightarrow q^{2}$, obtaining

$$
\sum_{i=1}^{l} C_{w_{i} \lambda} \sum_{j=1}^{k}\left(q^{-2\left(\rho, w_{i} \psi_{j}\right)}+q^{1+2\left(\rho, w_{i} \psi_{j}\right)}\right)=0 .
$$

Writing all the $C_{\mu}$ in their reduced form the recurrence can be rewritten as

$$
\sum_{\mu \leq \lambda} \Lambda_{\mu}^{\lambda, n}(q) C_{\mu}(q)=0
$$

for some coefficients $\Lambda_{\mu}^{\lambda, n}(q)$. Our purpose is to make more explicit these coefficients.

Remark 4.2 Set $\Omega_{\mu}^{\lambda, n}=\left\{w \lambda \mid w \in W / W_{\lambda}, w \lambda+\rho \sim \mu+\rho\right\}$. The sign change on the $n$-th coordinate $\epsilon_{n}$ induces a bijection between $\Omega_{\mu}^{2 \omega_{n-1}, n}$ and $\Omega_{\mu}^{2 \omega_{n}, n}$. Observe now that the general coefficient $\Lambda_{\mu}^{\lambda, n}(q)$ does not depend on the last coordinate of the weights in $\Omega_{\mu}^{\lambda, n}$ because the last coordinate of $\rho$ is equal to 0 . As a consequence $\Lambda_{\mu}^{2 \omega_{n-1}, n}(q)=\Lambda_{\mu}^{2 \omega_{n}, n}(q)$ for all $\mu \leq \lambda$ and then $C_{2 \omega_{n-1}}(q)=C_{2 \omega_{n}}(q)$. Coherently with the above notation, if $n$ is even we will denote the polynomial $C_{2 \omega_{n}}(q)$ by $C_{\frac{n}{2}}$.

Let us observe that, if $\lambda=\omega_{2 k}$ (resp. $\lambda=2 \omega_{n}$ and $\lambda=\omega_{n-1}+\omega_{n}$ ) then the non zero integral dominant weights smaller than $\lambda$ are of the form $\omega_{2 i}$ with $i<k$ (resp. $i<\lfloor n / 2\rfloor$ ). For brevity we will denote the coefficient $\Lambda_{\omega_{2 i}}^{\omega_{2 k}, n}(q)$ and the set $\Omega_{\omega_{2 i}}^{\omega_{2 k}, n}\left(\operatorname{resp} . \Lambda_{\omega_{2 i}}^{2 \omega_{n}, n}(q)\right.$ and $\Omega_{\omega_{2 i}}^{2 \omega_{n}, n}, \Lambda_{\omega_{2 i}}^{\omega_{n-1}+\omega_{n}, n}(q)$ and $\Omega_{\omega_{2 i}}^{\omega_{n-1}+\omega_{n}, n}$ ) by $\Lambda_{i}^{k, n}$ and $\Omega_{i}^{k, n}$ (resp. by $\Lambda_{i}^{\frac{n}{2}, n}$ and $\Omega_{i}^{\frac{n}{2}, n}$, $\Lambda_{i}^{\frac{n-1}{2}, n}$ and $\Omega_{i}^{\frac{n-1}{2}, n}$ ). It is immediate to show that the only contributions to the coefficient $\Lambda_{k}^{k, n}$ come from the case $w_{i}=i d$ and consequently $\Lambda_{k}^{k, n}$ is equal to

$$
\Lambda_{k}^{k, n}=\sum_{i=1}^{2 k} \frac{\left(1+q^{4(n-i)+1}\right)}{q^{2(n-i)}}=\frac{\left(q^{4 k}-1\right)\left(q^{4(n-k)-1}+1\right)}{q^{2(n-1)}\left(q^{2}-1\right)} .
$$

It is more difficult to obtain closed formulae for the generic $\Lambda_{h}^{k, n}$, however some nice recurrences hold.

Remark 4.3 A direct inspection shows that the weights giving non zero contribution to $\Lambda_{h}^{k, n}, k>h>0$ are of the form $e_{1}+\cdots+e_{2 h}+\mu$, where $\mu$ has the first $2 h$ coordinates equal to 0 . Considering the immersion of $D_{n-2 h} \rightarrow D_{n}$ induced by the Dynkin diagrams, this means that $\mu$ can be contracted to a weight in $\Omega_{0}^{k-h, n-2 h}$. By abuse of notation, we denote this contraction process writing $\mu \in \Omega_{0}^{k-h, n-2 h}$. 
As a consequence of the above Remark, the following relation holds: ${ }^{2}$

$$
\Lambda_{h}^{k, n}=(-1)^{k-h} \Lambda_{h}^{h, n}\left|\Omega_{0}^{k-h, n-2 h}\right|+\Lambda_{0}^{k-h, n-2 h} .
$$

We reduced to computing the coefficients $\Lambda_{0}^{k, n}$. For the weights conjugated to 0 some results similar to the ones proved in [10] for $B_{n}$ and $C_{n}$ hold:

Lemma 4.4 Let $n$ be even. Set $\lambda=2 \omega_{n}$ and let $w \in W$ be such that $w \lambda$ is conjugated to 0 .

(1) $w \lambda$ is equal to $(-1,1, \ldots,-1,1)$ if $n / 2$ is even and to $(-1,1, \ldots,-1,-1)$ if $n / 2$ is odd.

(2) There exists an element $\sigma \in W$ of $\operatorname{sign} \operatorname{sgn}(\sigma)=n / 2$ such that $\sigma(w \lambda+\rho)=\rho$.

Lemma 4.5 Set $\lambda=\omega_{2 k}, 2 k<n$ or $\lambda=\omega_{n-1}+\omega_{n}, n=2 k+1$ and let $w \in W$ be such that $w \lambda$ is conjugated to 0 . Then $w \lambda$ is of one of the following form:

(1) The $2 k$ non zero coordinates of $w \lambda$ are pair of consecutive coordinates $\left((w \lambda)_{(j)},(w \lambda)_{j+1}\right)$ of the form $(-1,1)$.

(2) There are $2(k-1)$ non zero coordinates that are pair of consecutive coordinates $\left((w \lambda)_{(j)},(w \lambda)_{j+1}\right)$ of the form $(-1,1)$ and the latter two are equal to -1 .

In both cases there exists an element $\sigma \in W$ of sign $\operatorname{sgn}(\sigma)=k$ such that $\sigma(w \lambda+\rho)=\rho$.

This produce an explicit formula for the number of weights in $\Omega_{0}^{\lambda, n}$ :

$$
\left|\Omega_{0}^{\lambda, n}\right|= \begin{cases}1 & \text { if } \lambda=2 \omega_{n} \text { and } n \text { is even or } \lambda=0 \\
\frac{n}{k}\left(\begin{array}{c}
n-k-1 \\
k-1
\end{array}\right) & \text { if } \lambda=\omega_{2 k} \text { and } 2 k<n \text { or } \lambda=\omega_{n-1}+\omega_{n} \text { and } n=2 k+1 .\end{cases}
$$

Furthermore, the coefficient $\Lambda_{0}^{\frac{n}{2}, n}$ can be easily computed. Set

$$
r(n, q)=q^{2(n-1)}-q^{-2(n-1)+1}+q^{-2(n-2)}+q^{2(n-2)+1}=(q+1)\left(q^{2 n-3}+q^{-2 n+3}\right)
$$

then

$$
\Lambda_{0}^{\frac{n}{2}, n}=(-1)^{\frac{n}{2}} \sum_{i=1}^{\frac{n}{2}} r(2 i, q)=(-1)^{\frac{n}{2}} r(n, q)-\Lambda_{0}^{\frac{n-2}{2}, n-2} .
$$

In the general case, producing explicit formulae for $\Lambda_{0}^{k, n}$ is more complicated. As a consequence of Lemma 4.5 we obtain a case by case analysis of the weights in $\Omega_{0}^{k, n}$ that leads us to some recursive expressions for $\Lambda_{0}^{k, n}$. Let $\mu$ be a weight in $\Omega_{0}^{k, n}$ :

Case 1: If $n=2 k+1$ then $\mu$ must be of the form $-e_{1}+e_{2}+v$ with $v \in \Omega_{0}^{k-1,2 k-1}$ or $\mu=\left(0, \mu_{2}, \ldots, \mu_{n}\right)$ where $\mu^{\prime}=\left(\mu_{2}, \ldots, \mu_{n}\right) \in \Omega_{0}^{k, 2 k}$ or $\epsilon_{n} \mu^{\prime} \in \Omega_{0}^{k, 2 k}$,

Case 2: If $n \neq 2 k, 2 k+1$ then $\mu$ must be of the form $-e_{1}+e_{2}+v$ with $v \in \Omega_{0}^{k-1, n-2}$ or $\mu=\left(0, \mu_{2}, \ldots, \mu_{n}\right)$ where $\mu^{\prime}=\left(\mu_{2}, \ldots, \mu_{n}\right) \in \Omega_{0}^{k, n-1}$.

We obtain the recursive relations:

$$
\begin{gathered}
\Lambda_{0}^{k, 2 k+1}=(-1)^{k} r(2 k+1, q)\left|\Omega_{0}^{k-1,2 k-1}\right|-\Lambda_{0}^{k-1,2 k-1}+2 \Lambda_{0}^{k, 2 k}, \\
\Lambda_{0}^{k, n}=(-1)^{k} r(n, q)\left|\Omega_{0}^{k-1, n-2}\right|-\Lambda_{0}^{k-1, n-2}+\Lambda_{0}^{k, n-1} .
\end{gathered}
$$

$\overline{{ }^{2} \text { Here we set by convention } \Omega_{0}^{1,2}}=\{(-1,-1)\}, \Omega_{0}^{1,3}=\{(-1,1,0),(0,-1,-1),(0,-1,1\}$ and coherently $\Lambda_{0}^{1,2}=r(2, q), \Lambda_{0}^{1,3}=r(3, q)+2 r(2, q)$ (c.f.r. Eq. 4.7) 
The above recurrences between the coefficients allow us to reduce the triangular system given by Stembridge's relations as described in the following Proposition, that we prove in Section 5. Set

$$
b_{k, n}= \begin{cases}\frac{\left(q^{4 k}-1\right)}{q^{2 k-1}(q-1)} & \text { if } n=2 k, \\ \frac{\left(q^{2 n}-1\right)\left(q^{2(n-2 k)}+1\right)}{q^{2(n-k)-1}(q-1)} & \text { otherwise. }\end{cases}
$$

Proposition 4.6 Let $R_{i}$ be the recurrence for $C_{i}$ written in reduced form. Then there exist a family of integers $\left\{A_{i}^{k, n}\right\}_{i \leq k}$ such that

$$
\sum_{i=1}^{k} A_{i}^{k, n} R_{i}=\Lambda_{k}^{k, n} C_{k}-\sum_{i=1}^{k} b_{i, n-2(k-i)} C_{k-i}
$$

Now, we apply an inductive reasoning. We recall that the base case of weight $\omega_{2}$ can be obtained comparing $P_{D}\left(V_{\omega_{2}}^{0}, q^{2}, q\right)$ with the formulae proved by Stembridge in [22]. Observe now that the following relations between the $b_{k, n}$ hold:

$$
\begin{gathered}
b_{i, n-2(k-i)}-b_{i-1, n-2(k-i)}=-\frac{(q+1)\left(q^{2(n-2 k+1)}-1\right)}{q^{2(n-2 k)+1}} \cdot \frac{\left(q^{2(n-2(k-i))}-1\right)}{q^{2 i}}, \\
2 b_{i, 2 i}-b_{i-1,2 i}=-\frac{(q+1)\left(q^{2}-1\right)}{q} \cdot \frac{\left(q^{4 i}-1\right)}{q^{2 i}} .
\end{gathered}
$$

If $k<\frac{n}{2}$, considering the difference between the recurrences of the form (4.10) for $C_{k}$ and $C_{k-1}$ we obtain:

$$
\begin{aligned}
\Lambda_{k}^{k, n} C_{k}-\left(b_{1, n-2(k-1)}+\Lambda_{k-1}^{k-1, n}\right) C_{k-1} & =\sum_{i=2}^{k}\left[b_{i, n-2(k-i)}-b_{i-1, n-2(k-i)}\right] C_{k-i} \\
& =-\frac{(q+1)\left(q^{2(n-2 k+1)}-1\right)}{q^{2(n-k)+1}} \sum_{i=0}^{k-2} q^{2 i}\left(q^{2(n-2 i)}-1\right) C_{i}
\end{aligned}
$$

Set

$$
\begin{gathered}
C(k, n, q)=\Lambda_{k}^{k, n} T_{k}^{n}-b_{1, n-2(k-1)}-\Lambda_{k-1}^{k-1, n}, \\
D(k, n, q)=-\frac{(q+1)\left(q^{2(n-2 k+1)}-1\right)}{q^{2(n-k)+1}} .
\end{gathered}
$$

The conjecture in this case is then reduced to prove that

$$
\frac{C(k, n, q)}{D(k, n, q)} C_{k-1}=\left[q^{2(k-2)}\left(q^{2(n-2(k-2))}-1\right)+\frac{C(k-1, n, q)}{D(k-1, n, q)}\right] C_{k-2} .
$$

and then to check a polynomial identity. This can be easily computed by a symbolic algebra software. We checked the above equality using SageMath [20]. In the case of $2 \omega_{n}$ with $n=2 k$, we have to consider 2 times (4.10) for $C_{\frac{n}{2}}$ minus the recurrence for $C_{k-1}$, obtaining

$$
\begin{aligned}
\Lambda_{k}^{k, n} 2 C_{n}-\left(2 b_{1,2}+\Lambda_{k-1}^{k-1, n}\right) C_{k-1} & =\sum_{i=2}^{k}\left[2 b_{i, 2 i}-b_{i-1,2 i}\right] C_{k-i} \\
& =-\frac{(q+1)\left(q^{2}-1\right)}{q^{n+1}} \sum_{i=0}^{k-2} q^{2 i}\left(q^{2(n-2 i)}-1\right) C_{i},
\end{aligned}
$$


and the computation is exactly the same as in the previous case. Observe that our choice of taking 2 times recurrence (4.10) for $C_{\frac{n}{2}}$ in the above computation is coherent with the Remark 4.1.

\subsection{Weights of the Form $\lambda=\omega_{1}+\omega_{2 k+1}, \lambda=\omega_{1}+\omega_{n-1}+\omega_{n}$ if $n$ is Even and $\lambda=\omega_{1}+2 \omega_{n-1}, \omega_{1}+2 \omega_{n}$ if $n$ is Odd}

We remark that in this case $W_{\lambda} \cdot e_{1}=\left\{e_{1}\right\}$ and then the minuscule recurrence becomes more explicit. We consider the recurrence (2.2) and make again the evaluations $q \rightarrow-q$ and $t \rightarrow q^{2}$, obtaining

$$
\sum_{i=1}^{l} C_{w_{i} \lambda} \frac{\left(1-q^{1+4\left(\rho, w_{i} e_{1}\right)}\right)}{q^{2\left(\rho, w_{i} e_{1}\right)}}=0 .
$$

The recurrence (4.12) can be written in reduced form as:

$$
R_{k}: \sum_{i=0}^{k} C_{2 \mid i} \Lambda_{2 \mid i}^{2 \mid k, n}+\sum_{i=0}^{k+1 / k} C_{i} \Lambda_{i}^{2 \mid k, n}=0,
$$

for some coefficients $\Lambda_{2 \mid i}^{2 \mid k, n}$ and $\Lambda_{i}^{2 \mid k, n}$ that we are going now to analyze more closely. We underline that the index of the second sum goes from 0 to $k$ if $n=2 k+1$ and to $k+1$ if $n>2 k+1$. In the special case $n=2 k+2$ the recurrence can be displayed as follows:

$$
R_{k}: \sum_{i=0}^{k} C_{2 \mid i} \Lambda_{2 \mid i}^{2 \mid k, n}+\sum_{i=0}^{k} C_{i} \Lambda_{i}^{2 \mid k, n}+C_{k+1}\left[\Lambda_{2 \omega_{n}}^{2 \mid k, n}+\Lambda_{2 \omega_{n-1}}^{2 \mid k, n}\right]=0 .
$$

Using an argument similar to Remark 4.2 we have $\Lambda_{2 \omega_{n}}^{2 \mid k, n}=\Lambda_{2 \omega_{n-1}}^{2 \mid k, n}$ and then the recurrence becomes:

$$
R_{k}: \sum_{i=0}^{k} C_{2 \mid i} \Lambda_{2 \mid i}^{2 \mid k, n}+\sum_{i=0}^{k} C_{i} \Lambda_{i}^{2 \mid k, n}+2 C_{n} \Lambda_{k+1}^{2 \mid k, n}=0 .
$$

Moreover, by the same reasoning of Remark 4.2, if $n=2 k+1$ we obtain that $C_{\omega_{1}+2 \omega_{n-1}}(q)=C_{\omega_{1}+2 \omega_{n}}(q)$ and then we reduce to computing only $C_{\omega_{1}+2 \omega_{n}}(q)$ (that for brevity we will denote by $\left.C_{2 \mid k, 2 k+1}\right)$. As in the previous case we want to determine closed formulae and recursive relations for the coefficients that allow us to reduce the system.

Remark 4.7 Similarly to what we observed in the case $\omega_{2 k}$, the only contributions to $\Lambda_{2 \mid h}^{2 \mid k, n}$ come from weights of the form $2 e_{1}+e_{2}+\cdots+e_{2 h+1}+\mu$ with $\mu \in \Omega_{0}^{k-h, n-2 h-1}$.

Set

$$
s(n, q)=\frac{\left(1-q^{4 n+2}\right)}{q^{2 n}}
$$

by previous Remark we obtain:

$$
\Lambda_{2 \mid h}^{2 \mid k, n}=(-1)^{k-h} s(n-1, q)\left|\Omega_{0}^{k-h, n-2 h-1}\right| .
$$

Coherently with the notation of the previous section, we will denote by $\Omega_{2 \mid h}^{2 \mid k, n}$ (resp. $\Omega_{h}^{2 \mid k, n}$ ) the set $\Omega_{\omega_{1}+\omega_{2 h+1}}^{\omega_{1}+\omega_{2 k+1}, n}$ (resp. $\Omega_{\omega_{2 h}}^{\omega_{1}+\omega_{2 k+1}, n}$ ). It is immediate to show that the weights in $\Omega_{k+1}^{2 \mid k, n}$ 
are all of the form $e_{1}+\cdots+e_{j-2}+2 e_{j}+\cdots+e_{2(k+1)}$ for $j \leq 2(k+1)$. As a consequence, we obtain the following formula:

$$
\Lambda_{k+1}^{2 \mid k, n}=-\sum_{j=2}^{2 k+2} s(n-j, q)=-\frac{\left(q^{4(n-k-1)-2}-1\right)\left(q^{4(k+1)-2}-1\right)}{q^{2(n-2)}\left(q^{2}-1\right)} .
$$

Remark 4.8 (Reeder's Conjecture for $2 \omega_{1}$ ) Formulae (4.13) and (4.14) describe explicitly the coefficients $\Lambda_{2 \mid 0}^{2 \mid 0, n}$ and $\Lambda_{1}^{2 \mid 0, n}$. By direct inspection it is possible to prove that $\Omega_{0}^{2 \mid 0, n}=$ $\left\{-2 e_{n-1}\right\}$ and consequently $\Lambda_{0}^{2 \mid 0, n}=s(-1, q)=-s(0, q)$. Now $R_{0}$ is explicit and the Reeder's Conjecture in this case can be proved comparing the obtained expression for $C_{2 \mid 0, n}$ with the formula for $P_{D}\left(\tilde{\pi}_{(n-1,1), \emptyset} ; q^{2}, q\right) .^{3}$

To find a recursive expansion of the coefficients $\Lambda_{h}^{2 \mid k, n}$ for $k \geq 1$ we have to consider the cases $n=2 k+1,2 k+2$ and $n$ generic in three different ways. Firstly suppose $k \geq h>1$. If $n=2 k+1$ a weight $\mu \in \Omega_{h}^{2 \mid k, n}$ must be of the form $\omega_{2}+\mu^{\prime}$ with $\mu^{\prime} \in \Omega_{h-1}^{2 \mid k-1, n-2}$, obtaining

$$
\Lambda_{h}^{2 \mid k, n}=\Lambda_{h-1}^{2 \mid k-1,2(k-1)+1} .
$$

The case $n=2 k+2$ needs a closer analysis. A weight $\mu \in \Omega_{h}^{2 \mid k, n}$ can be of one of the following forms:

Case $1 \mu=e_{1}+e_{2}+\mu^{\prime}$ with $\mu^{\prime} \in \Omega_{h-1}^{2 \mid k-1, n-2}$,

Case $2 \mu=2 e_{2}+\mu^{\prime}$ with $\mu^{\prime} \in \Omega_{h-1}^{k, n-2} \cup \epsilon_{n} \Omega_{h-1}^{k, n-2}$ or equivalently $\mu_{1}=0$ and $\tilde{\mu}=$ $\left(\mu_{2}, \ldots, \mu_{n}\right) \in \Omega_{2 \mid h-1}^{2 \mid k, n-1} \cup \epsilon_{n} \Omega_{2 \mid h-1}^{2 \mid k, n-1}$,

Case $3 \mu=\left(1,0,2,1, \mu_{5}, \ldots, \mu_{n}\right)$ where $\tilde{\mu}=\left(\mu_{5}, \ldots, \mu_{n}\right) \in \Omega_{h-2}^{k-1,2 k-2} \cup$ $\epsilon_{n} \Omega_{h-2}^{k-1,2 k-2}$.

This analysis can be translated in the following relation:

$$
\Lambda_{h}^{2 \mid k, 2 k+2}=\Lambda_{h-1}^{2 \mid k-1,2 k}-2 \Lambda_{2 \mid h-1}^{2 \mid k, 2 k+1}-2(-1)^{k-h+1} s(2 k-1, q) .
$$

The case of $n$ generic is completely analogous to the previous one, except for the fact that $\Omega_{h-1}^{k, n-2} \cup \epsilon_{n} \Omega_{h-1}^{k, n-2}=\Omega_{h-1}^{k, n-2}$ and $\Omega_{h-2}^{k-1, n-4} \cup \epsilon_{n} \Omega_{h-2}^{k-1, n-4}=\Omega_{h-2}^{k-1, n-4}$ leading to the recurrence:

$$
\Lambda_{h}^{2 \mid k, n}=\Lambda_{h-1}^{2 \mid k-1, n-2}-\Lambda_{2 \mid h-1}^{2 \mid k, n-1}-(-1)^{k-h+1} s(n-3, q)\left|\Omega_{0}^{k-h+1, n-2 h}\right| .
$$

The case $h=1$ must be considered differently. If $n=2 k+1$, the elements in $\Omega_{1}^{k, 2 k+1}$ are of the form:

Case $1 \quad \mu=\omega_{2}+\mu^{\prime}$ with $\mu^{\prime} \in \Omega_{0}^{2 \mid k-1, n-2}$,

Case $2 \mu=\left(1,-1,2, \mu_{4}, \ldots, \mu_{n}\right)$ with $\tilde{\mu}=\left(\mu_{4}, \ldots, \mu_{n}\right) \in \Omega_{0}^{k-1, n-3}$,

\footnotetext{
${ }^{3}$ Observe that, to prove inductively the Conjecture, it is also needed to check explicitly the cases $\omega_{1}+\omega_{3}$ in $D_{4}$ and $\omega_{1}+\omega_{5}$ in $D_{5}$. In these cases to list directly the weights involved in the computation of coefficients is quite simple. It is possible to achieve a description coherent with the general analysis in the paper setting by convention $\Omega_{0}^{2 \mid 0, n}=\{(-2,0)\}$ for every $n>1$ and $\Omega_{0}^{2 \mid 1,3}=\{(-1,-1,2),(-2,1,-1)\}, \Omega_{1}^{2 \mid 1,3}=$ $\{(1,-1,2)\}$. Coherently $\Lambda_{0}^{2 \mid 1,3}=s(0, q)+s(-2, q)$ and $\Lambda_{h-1}^{2 \mid k-1,2(k-1)+1}=-s(0, q)$.
} 
obtaining the recurrence:

$$
\Lambda_{1}^{2 \mid k, 2 k+1}=\Lambda_{0}^{2 \mid k-1,2 k-1}-(-1)^{k-1} s(2 k-2, q) .
$$

In the other cases we must consider a third family of weights, i.e. of the form $\left(0, \mu_{2}, \ldots, \mu_{n}\right)$ where $\tilde{\mu}=\left(\mu_{2}, \ldots, \mu_{n}\right)$ is contained in $\Omega_{2 \mid 0}^{2 \mid k, 2 k+1} \cup \epsilon_{n} \Omega_{2 \mid 0}^{2 \mid k, 2 k+1}$ if $n=$ $2 k+2$ and in $\Omega_{2 \mid 0}^{2 \mid k, n-1}$ for $n$ generic. Consequently we obtain:

$$
\begin{aligned}
\Lambda_{1}^{2 \mid k, 2 k+2} & =\Lambda_{0}^{2 \mid k-1,2 k}-2 \Lambda_{2 \mid 0}^{2 \mid k, 2 k+1}-(-1)^{k-1} s(2 k-1, q)\left|\Omega_{0}^{k-1,2 k-1}\right|, \\
\Lambda_{1}^{2 \mid k, n} & =\Lambda_{0}^{2 \mid k-1, n-2}-\Lambda_{2 \mid 0}^{2 \mid k, n-1}-(-1)^{k-1} s(n-3, q)\left|\Omega_{0}^{k-1, n-3}\right| .
\end{aligned}
$$

Finally, we will find recurrences for coefficient $\Lambda_{0}^{2 \mid k, n}$. Again, let us start from $n=2 k+1$. The only relevant cases are:

Case $1 \mu=\left(-1,1, \mu_{3}, \ldots, \mu_{n}\right)$ with $\tilde{\mu}=\left(\mu_{3}, \ldots, \mu_{n}\right) \in \Omega_{0}^{2 \mid k-1, n-2}$,

Case $2 \mu=\left(-1,-1,2, \mu_{4}, \ldots, \mu_{n}\right)$ with $\tilde{\mu}=\left(\mu_{4}, \ldots, \mu_{n}\right) \in \Omega_{0}^{k-1, n-3}$,

Case $3 \mu=\left(-2,1,1, \mu_{4}, \ldots, \mu_{n}\right)$ with $\tilde{\mu}=\left(\mu_{4}, \ldots, \mu_{n}\right) \in \Omega_{0}^{k-1, n-3}$,

and consequently we obtain:

$$
\Lambda_{0}^{2 \mid k, 2 k+1}=-\Lambda_{0}^{2 \mid k-1,2 k-1}+(-1)^{k-1}[s(2 k-2, q)+s(-2 k, q)] .
$$

As in the case $h=1$, if $n \neq 2 k+1$ we have to add to the previous list the weights of the form $\left(0, \mu_{2}, \ldots, \mu_{n}\right)$ with $\tilde{\mu}=\left(\mu_{2}, \ldots, \mu_{n}\right)$ contained respectively in $\Omega_{0}^{2 \mid k, 2 k+1} \cup \epsilon_{n} \Omega_{0}^{2 \mid k, 2 k+1}$ if $n=2 k+2$ and in $\Omega_{0}^{2 \mid k, n-1}$ for $n$ generic. This yields to the recurrences:

$$
\begin{aligned}
\Lambda_{0}^{2 \mid k, 2 k+2} & =2 \Lambda_{0}^{2 \mid k, 2 k+1}-\Lambda_{0}^{2 \mid k-1,2 k}+(-1)^{k-1}[s(2 k-1, q)+s(-2 k-1, q)]\left|\Omega_{0}^{k-1,2 k-1}\right|, \\
\Lambda_{0}^{2 \mid k, n} & =\Lambda_{0}^{2 \mid k, n-1}-\Lambda_{0}^{2 \mid k-1, n-2}+(-1)^{k-1}[s(n-3, q)+s(-n+1, q)]\left|\Omega_{0}^{k-1, n-3}\right| .
\end{aligned}
$$

Set now

$$
d_{k, n}:=s(k, q)+s(n-k-1, q)=\frac{q^{2 k}\left(1-q^{2 n}\right)\left(1+q^{2(n-2 k-1)}\right)}{q^{2(n-1)}},
$$

imposing that the coefficients $\Lambda_{2 \mid h}^{2 \mid k, n}$ must cancel, we are again able to find a nice reduction of the triangular system, proved in Section 5.

Proposition 4.9 Let $R_{i}$ be the reduced recurrence for $C_{2 \mid i}$. Then there exist a family of integers $\left\{E_{i}^{k, n}\right\}_{i \leq k}$, with $E_{k}^{k, n}=1$ and $\sum_{i=h}^{k}(-1)^{i}\left(\begin{array}{c}n-i-1 \\ i\end{array}\right) E_{i}^{k n}=0$, such that

$$
\sum_{i=0}^{k} E_{i}^{k, 2 k+1} R_{i}=\Lambda_{2 \mid k}^{2 \mid k, 2 k+1} C_{2 \mid k, 2 k+1}+\Gamma_{k}^{2 \mid k, 2 k+1} C_{k, 2 k+1}-\sum_{j=0}^{k-1} s(k-j, q) C_{j, 2 k+1},
$$

and

$$
\sum_{i=0}^{k} E_{i}^{k, n} R_{i}=\Lambda_{2 \mid k}^{2 \mid k, n} C_{2 \mid k, n}+\Gamma_{k+1}^{2 \mid k, n} C_{k+1, n}+\Gamma_{k}^{2 \mid k, n} C_{k, n}-\sum_{j=0}^{k-1} d_{k-j, n-2 j} C_{j, n} .
$$

for some suitable coefficients $\Gamma_{k}^{2 \mid k, 2 k+1}, \Gamma_{k+1}^{2 \mid k, n}$ and $\Gamma_{k}^{2 \mid k, n}$. 
We remark that it is possible to find a recursive expression similar to Eq. 4.14 for the coefficient $\Gamma_{k}^{2 \mid k, n}$ (c.f.r. Section 5, Proposition 5.4, computations for $\Gamma_{h}^{2 \mid k, n}$ ):

$$
\begin{gathered}
\Gamma_{1}^{2 \mid 1, n}=-s(0, q)-s(n-2, q)-s(n-3, q) \\
\Gamma_{k}^{2 \mid k, n}=\Gamma_{k-1}^{2 \mid k-1, n-2}-[s(n-2, q)+s(n-3, q)]
\end{gathered}
$$

A direct inspection shows that $\Gamma_{2}^{2 \mid 2,5}=-\sum_{j=0}^{3} s(j, q)$ and, starting from the formula for $\Gamma_{1}^{2 \mid 1, n}$, it is possible to obtain the following expressions:

$\Gamma_{k}^{2 \mid k, 2 k+1}=-\sum_{j=0}^{2 k-1} s(j, n)=-\frac{\left(q^{4 k}-1\right)^{2}}{q^{4 k-2}\left(q^{2}-1\right)}, \quad \Gamma_{k}^{2 \mid k, n}=-s(0, q)+s(n-2 k-2, q)+\Gamma_{k+1}^{2 \mid k, n}$.

\subsection{Reeder's Conjecture for $\lambda=\omega_{1}+\omega_{2 k+1}, \lambda=\omega_{1}+\omega_{n-1}+\omega_{n}$ if $n$ is Even} and $\lambda=\omega_{1}+2 \omega_{n-1}, \omega_{1}+2 \omega_{n}$ if $n$ is Odd

We recall that in Section 4.1 we shown the identity:

$$
\frac{C(k, n, q)}{D(k, n, q)} C_{k-1}=\sum_{i=0}^{k-2} q^{2 i}\left(q^{2(n-2 i)}-1\right) C_{i} .
$$

Substituting Eq. 4.17 in Eq. 4.15 we obtain

$$
\begin{aligned}
\Lambda_{2 \mid k}^{2 \mid k, 2 k+1} C_{2 \mid k, 2 k+1} & =-\Gamma_{k}^{2 \mid k, 2 k+1} C_{k, 2 k+1}+\sum_{j=0}^{k-1} s(k-j, q) C_{j, 2 k+1} \\
& =-\Gamma_{k}^{2 \mid k, 2 k+1} C_{k, 2 k+1}-\sum_{j=0}^{k-1} \frac{q^{2 j}\left(q^{2(2 k+1-2 j)}-1\right)}{q^{2 k}} C_{j, 2 k+1} \\
& =-\Gamma_{k}^{2 \mid k, 2 k+1} C_{k, 2 k+1}-\frac{C_{k-1,2 k+1}}{q^{2 k}}\left[q^{2(k-1)}\left(q^{6}-1\right)+\frac{C(k, n, q)}{D(k, n, q)}\right] \\
& =-\frac{C_{k-1,2 k+1}}{q^{2 k}}\left[q^{2 k} \Gamma_{k}^{2 \mid k, 2 k+1} T_{k}^{2 k+1}+q^{2(k-1)}\left(q^{6}-1\right)+\frac{C(k, n, q)}{D(k, n, q)}\right],
\end{aligned}
$$

and analogously, substituting Eq. 4.17 in Eq. 4.16

$$
\begin{aligned}
\Lambda_{2 \mid k}^{2 \mid k, n} C_{2 \mid k, n} & =-\Gamma_{k+1}^{2 \mid k, n} C_{k+1, n}-\Gamma_{k}^{2 \mid k, n} C_{k, n}+\sum_{j=0}^{k-1} d_{k-j, n-2 j} C_{j, n} \\
& =-\Gamma_{k+1}^{2 \mid k, n} C_{k+1, n}-\Gamma_{k}^{2 \mid k, n} C_{k, n}+\frac{\left(q^{2(n-2 k-1)}+1\right)}{q^{2(n-k-1)}} \sum_{j=0}^{k-1} q^{2 j}\left(q^{2(n-2 j)}-1\right) C_{j, n} \\
& =-C_{k, n}\left[\Gamma_{k+1}^{2 \mid k, n} T_{k+1}^{n}+\Gamma_{k}^{2 \mid k, n}+\frac{\left(q^{2(n-2 k-1)}+1\right)}{q^{2(n-k-1)}} \frac{C(k, n, q)}{D(k, n, q)}\right] .
\end{aligned}
$$

Again proving Reeder's Conjecture is equivalent to a polynomial identity that we checked with SageMath [20]. 


\section{Proof of Propositions 4.5 and 4.7}

In this Section we denote by $\Theta(n . k, h)$ the quantity $\left|\Omega_{h}^{k, n}\right|=\left|\Omega_{0}^{k-h, n-2 h}\right|$.

Remark 5.1

$$
\begin{gathered}
\Theta(n, k, h)=\Theta(n, k, h+1)+\Theta(n-1, k, h), \\
\Theta(2 k+1, k, h)=\Theta(2 k+1, k, h+1)+2 \Theta(2 k, k, h) .
\end{gathered}
$$

Set now

$$
A_{h}^{k, n}= \begin{cases}0 & \text { if } h>k \text { or } h \leq 0, \\ 1 & \text { if } h=k \\ -\sum_{i=h+1}^{k}(-1)^{i-h} \Theta(n, i, h) A_{i}^{k, n} & \text { otherwise. }\end{cases}
$$

Proposition 5.2 Let $R_{i}$ be the recurrence for $C_{i}$ written in reduced form. Then

$$
\sum_{i=1}^{k} A_{i}^{k, n} R_{i}=\Lambda_{k}^{k, n} C_{k}-\sum_{i=0}^{k-1} b_{k-i, n-2 i} C_{i}
$$

Proof We will use $\Gamma_{h}^{k, n}$ to denote the coefficient of $C_{h}$ in $\sum_{i=1}^{k} A_{i}^{k, n} R_{i}$. Using Remark 5.1 and Definition (5.1) it is possible to prove that the integers of the form $A_{h}^{k, n}$ satisfy some nice iterative properties:

Lemma 5.3 (1) $A_{h}^{k, n}=A_{h-1}^{k-1, n-2}$ for $h>1$,

(2) $A_{h}^{k, n}=A_{h}^{k, n-1}+A_{h}^{k-1, n-1}$ if $n \neq 2 k, 2 k+1$,

(3) $A_{h}^{k, 2 k}=A_{h}^{k-1,2 k-1}$,

(4) $A_{h}^{k, 2 k+1}=2 A_{h}^{k, 2 k}+A_{h}^{k-1,2 k}$.

Using (1) it is easy to prove that $\Gamma_{h}^{k, n}=\Gamma_{0}^{k-h, n-2 h}$ if $k>h>0$ :

$$
\begin{aligned}
\Gamma_{h}^{k, n} & =\sum_{j=h}^{k} A_{j}^{k, n} \Lambda_{h}^{j, n} \\
& =\left[\sum_{j=h}^{k}(-1)^{j-h} \Theta(n, j, h) A_{j}^{k, n}\right] \Lambda_{h}^{h, n}+\sum_{j=h+1}^{k} A_{j}^{k, n} \Lambda_{0}^{j-h, n-2 h} \\
& =\sum_{t=1}^{k-h} A_{t}^{k-h, n-2 h} \Lambda_{0}^{t, n-2 h}=\Gamma_{0}^{k-h, n-2 h} .
\end{aligned}
$$

To recover the coefficients of Eq. 5.2 we have to compute $\Gamma_{0}^{k, n}$. In particular we want to prove by inductive reasoning that

$$
\Gamma_{0}^{k, 2 k}=-\sum_{j=2}^{k+1} r(j, q), \quad \Gamma_{0}^{k, 2 k+1}=2 \Gamma_{0}^{k, 2 k}-r(k+2, q), \quad \Gamma_{0}^{k, n}=\Gamma_{0}^{k, n-1}-r(n-k+1, q) .
$$


The formula for $\Gamma_{0}^{2,4}$ can be easily checked by direct computations. We have to distinguish three different cases. If $n=2 k$ and using (1) and (3) of Lemma 5.3 we have:

$$
\begin{aligned}
\Gamma_{0}^{k, 2 k} & =\sum_{j=1}^{k}\left[\Lambda_{0}^{j, n-1}-\Lambda_{0}^{j-1, n-2}\right] A_{j}^{k . n}+\sum_{j=1}^{k}\left[(-1)^{j} \Theta(n, j, 1) A_{j}^{k, n}\right] r(2 k, q) \\
& =\sum_{j=1}^{k-1} A_{j}^{k-1, n-1} \Lambda_{0}^{j, n-1}-\sum_{t=1}^{k-1} A_{t}^{k-1, n-2} \Lambda_{0}^{t, n-2} \\
& =\Gamma_{0}^{k-1,2(k-1)+1}-\Gamma_{0}^{k-1,2(k-1)}={ }^{\text {Ind }} \Gamma_{0}^{k-1,2(k-1)}-r(k+1, q) .
\end{aligned}
$$

The computation in the case $n=2 k+1$ needs the additional use of (4).

$$
\begin{aligned}
\Gamma_{0}^{k, 2 k+1}= & \sum_{j=1}^{k-1}\left[\Lambda_{0}^{j, n-1}-\Lambda_{0}^{j-1, n-2}\right] A_{j}^{k, n}+\sum_{j=1}^{k}\left[(-1)^{j} \Theta(n, j, 1) A_{j}^{k, n}\right] r(2 k+1, q) \\
& +2 \Lambda_{0}^{k, 2 k}-\Lambda_{0}^{k-1,2 k-1} \\
= & \sum_{j=1}^{k-1} A_{j}^{k, n} \Lambda_{0}^{j, 2 k}+2 \Lambda_{0}^{k, 2 k}-\sum_{t=1}^{k-1} A_{t}^{k-1, n-2} \Lambda_{0}^{t, n-2} \\
= & \sum_{j=1}^{k-1}\left[A_{j}^{k-1,2 k}+2 A_{j}^{k, 2 k}\right] \Lambda_{0}^{j, 2 k}+2 \Lambda_{0}^{k, 2 k}-\Gamma_{0}^{k-1, n-2} \\
= & \Gamma_{0}^{k-1,2 k}+2 \Gamma_{0}^{k, 2 k}-\Gamma_{0}^{k-1, n-2} \\
= & \text { Ind } 2 \Gamma_{0}^{k, 2 k)}-r(k+2, q) .
\end{aligned}
$$

The case of $n$ generic is completely analogous and uses (2) of Lemma 5.3 instead of (3).

$$
\begin{aligned}
\Gamma_{0}^{k, n} & =\sum_{j=1}^{k}\left[\Lambda_{0}^{j, n-1}-\Lambda_{0}^{j-1, n-2}\right] A_{j}^{k, n}+\sum_{j=1}^{k}\left[(-1)^{j} \Theta(n, j, 1) A_{j}^{k, n}\right] r(n, q) \\
& =\sum_{j=1}^{k}\left[A_{j}^{k-1, n-1}+A_{j}^{k, n-1}\right] \Lambda_{0}^{j, n-1}-\Gamma_{0}^{k-1, n-2} \\
& =\Gamma_{0}^{k-1, n-1}+\Gamma_{0}^{k, n-1}-\Gamma_{0}^{k-1, n-2} \\
& ={ }^{I n d} \Gamma_{0}^{k, n-1}-r(n-k+1, q) .
\end{aligned}
$$

Now it is straightforward to show that $\Gamma_{0}^{k, n}=b_{k, n}$ and then $\Gamma_{h}^{k, n}=\Gamma_{0}^{k-h, n-2 k}=b_{k-h, n-2 h}$.

Set now $\Phi(n, k, h)=\Theta(n-1, k, h)$ and define

$$
E_{h}^{k, n}= \begin{cases}0 & \text { if } h>k \text { or } h<0 \\ 1 & \text { if } h=k \\ -\sum_{i=h+1}^{k}(-1)^{i-h} \Phi(n, i, h) E_{i}^{k, n} & \text { otherwise }\end{cases}
$$


Proposition 5.4 Let $R_{i}$ be the reduced recurrence for $C_{2 \mid i}$. Then

$$
\begin{aligned}
\sum E_{i}^{k, 2 k+1} R_{i}= & \Lambda_{2 \mid k}^{2 \mid k, 2 k+1} C_{2 \mid k, 2 k+1}+\Gamma_{k}^{2 \mid k, 2 k+1} C_{k, 2 k+1} \\
& -\sum_{j=0}^{k-1} s(k-j, q) C_{j, 2 k+1}, \\
\sum E_{i}^{k, n} R_{i}= & \Lambda_{2 \mid k}^{2 \mid k, n} C_{2 \mid k, n}+\Gamma_{k+1}^{2 \mid k, n} C_{k+1, n}+\Gamma_{k}^{k, n} C_{2 \mid k, n} \\
& -\sum_{j=0}^{k-1} d_{k-j, n-2 j} C_{j, n} .
\end{aligned}
$$

Proof Coherently with notation of Section 4, we will denote by $\Gamma_{2 \mid h}^{2 \mid k, n}\left(\operatorname{resp} \Gamma_{h}^{2 \mid k, n}\right.$ ) the coefficient of $C_{2 \mid h}$ (resp. $C_{h}$ ) in $\sum E_{i}^{k, 2 k+1} R_{i}$. Observing that $E_{h}^{k, n}=A_{h}^{k, n-1}$ we obtain an analogous of Lemma 5.3:

Lemma 5.5 (1) $E_{h}^{k, n}=E_{h-1}^{k-1, n-2}$ for $h>1$,

(2) $E_{h}^{k, n}=E_{h}^{k, n-1}+E_{h}^{k-1, n-1}$ if $n \neq 2 k+1,2 k+2$,

(3) $E_{h}^{k, 2 k+1}=E_{h}^{k-1,2 k}$,

(4) $E_{h}^{k, 2 k+2}=2 E_{h}^{k, 2 k+1}+E_{h}^{k-1,2 k+1}$.

An immediate consequence of Definition 5.3 is that $\Gamma_{2 \mid h}^{2 \mid k, n}=0$ if $h<k$. If $k>h>0$, we have:

$$
\begin{aligned}
\Gamma_{h}^{2 \mid k, 2 k+1}= & \sum_{j=h-1}^{k} E_{j}^{k, 2 k+1} \Lambda_{h}^{2 \mid j, n} \\
= & \sum_{j=h-1}^{k} E_{j}^{k, 2 k+1} \Lambda_{h-1}^{2 \mid j-1,2 k-1}-\sum_{j=h-1}^{k-1} E_{j}^{k, 2 k+1} \Lambda_{2 \mid h-1}^{2 \mid j, 2 k}-s(2 k-2, q) \\
& \times\left[\sum_{j=h-1}^{k-1}(-1)^{j-h+1} E_{j}^{k, 2 k+1} \Phi(2 k, j, h-1)\right] \\
= & \sum_{j=h-1}^{k} E_{j}^{k, 2 k+1} \Lambda_{h-1}^{2 \mid j-1,2 k-1}-\sum_{j=h-1}^{k-1} E_{j}^{k-1,2 k} \Lambda_{2 \mid h-1}^{2 \mid j, 2 k}-s(2 k-2, q) \\
& \times\left[\sum_{j=h-1}^{k-1}(-1)^{j-h+1} E_{j}^{k-1,2 k} \Phi(2 k, j, h-1)\right] \\
= & \Gamma_{h-1}^{2 \mid k-1,2 k-1},
\end{aligned}
$$




$$
\begin{aligned}
& \Gamma_{h}^{2 \mid k, 2 k+2}=\sum_{j=h-1}^{k} E_{j}^{k, 2 k+2} \Lambda_{h-1}^{2 \mid j-1,2 k}-2 \Lambda_{2 \mid h-1}^{2 \mid k, 2 k+1}-\sum_{j=h-1}^{k-1} E_{j}^{k, 2 k+2} \Lambda_{2 \mid h-1}^{2 \mid j, 2 k+1}+ \\
& -s(2 k-1, q)\left[2(-1)^{k-h+1} \Phi(2 k+1, k, h-1)\right. \\
& \left.+\sum_{j=h-1}^{k-1}(-1)^{j-h+1} E_{j}^{k, 2 k+2} \Phi(2 k+1, j, h-1)\right] \\
& =\Gamma_{h-1}^{2 \mid k-1,2 k}-\sum_{j=h-1}^{k}\left[2 E_{j}^{k, 2 k+1}+E_{j}^{k-1,2 k+1}\right] \Lambda_{2 \mid h-1}^{2 \mid j, 2 k+1}+ \\
& -s(2 k-1, q)\left[\sum_{j=h-1}^{k}(-1)^{j-h+1}\left(2 E_{j}^{k, 2 k+1}+E_{j}^{k-1,2 k+1}\right) \Phi(2 k+1, j, h-1)\right] \\
& =\Gamma_{h-1}^{2 \mid k-1,2 k}-2 \sum_{j=h-1}^{k} E_{j}^{k, 2 k+1} \Lambda_{2 \mid h-1}^{2 \mid j, 2 k+1}-\sum_{j=h-1}^{k-1} E_{j}^{k-1, n-1} \Lambda_{2 \mid h-1}^{2 \mid j, n-1} \\
& =\Gamma_{h-1}^{2 \mid k-1,2 k}, \\
& \Gamma_{h}^{2 \mid k, n}=\sum_{j=h-1}^{k} E_{j}^{k, n} \Lambda_{h-1}^{2 \mid j-1, n-2}-\sum_{j=h-1}^{k} E_{j}^{k, n} \Lambda_{2 \mid h-1}^{2 \mid j, n-1}-s(n-3, q) \\
& \times\left[\sum_{j=h-1}^{k}(-1)^{j-h+1} E_{j}^{k, n} \Phi(n-1, j, h-1)\right] \\
& =\Gamma_{h-1}^{2 \mid k-1, n-2}-\sum_{j=h-1}^{k}\left[E_{j}^{k, n-1}+E_{j}^{k-1, n-1}\right] \Lambda_{2 \mid h-1}^{2 \mid j, n-1} \\
& =\Gamma_{h-1}^{2 \mid k-1, n-2}-\sum_{j=h-1}^{k} E_{j}^{k, n-1} \Lambda_{2 \mid h-1}^{2 \mid j, n-1}-\sum_{j=h-1}^{k-1} E_{j}^{k-1, n-1} \Lambda_{2 \mid h-1}^{2 \mid j, n-1} \\
& =\Gamma_{h-1}^{2 \mid k-1, n-2} .
\end{aligned}
$$

We reduced again to the computation of explicit formulae for the zero coefficient in the recurrences.

$$
\begin{aligned}
\Gamma_{0}^{2 \mid k, 2 k+1} & =\sum_{j=0}^{k} E_{j}^{k, 2 k+1} \Lambda_{0}^{2 \mid j, n} \\
& =\sum_{j=0}^{k-1} E_{j}^{k, 2 k+1} \Lambda_{0}^{2 \mid j, 2 k}-\sum_{j=1}^{k} E_{j}^{k, 2 k+1} \Lambda_{0}^{2 \mid j-1,2 k-1}
\end{aligned}
$$




$$
\begin{aligned}
& +(s(2 k-2, q)+s(-2 k, q))\left[\sum_{j=1}^{k}(-1)^{j-1} E_{j}^{k, 2 k+1} \Phi(2 k+1, j, 1)\right] \\
= & \sum_{j=0}^{k-1} E_{j}^{k-1,2 k} \Lambda_{0}^{2 \mid j, 2 k}-\Gamma_{0}^{2 \mid k-1,2 k-1} \\
= & \Gamma_{0}^{2 \mid k-1,2 k}-\Gamma_{0}^{2 \mid k-1,2 k-1}, \\
\Gamma_{0}^{2 \mid k, 2 k+2}= & 2 \Lambda_{0}^{2 \mid k, 2 k+1}+\sum_{j=0}^{k-1} E_{j}^{k, 2 k+2} \Lambda_{0}^{2 \mid j, 2 k+1}-\sum_{j=1}^{k} E_{j}^{k, 2 k+2} \Lambda_{0}^{2 \mid j-1,2 k} \\
& +(s(2 k-1, q)+s(-2 k-1, q))\left[\sum_{j=1}^{k}(-1)^{j-1} E_{j}^{k, 2 k+2} \Phi(2 k+2, j, 1)\right. \\
= & 2 \Lambda_{0}^{2 \mid k, 2 k+1}+\sum_{j=0}^{k-1}\left[2 E_{j}^{k, 2 k+1}+E_{j}^{k-1,2 k+1}\right] \Lambda_{0}^{2 \mid j, 2 k+1}-\Gamma_{0}^{2 \mid k-1,2 k} \\
= & 2 \Gamma_{0}^{2 \mid k, 2 k+1}+\Gamma_{0}^{2 \mid k-1,2 k+1}-\Gamma_{0}^{2 \mid k-1,2 k}, \\
= & \sum_{j=0}^{k-1}\left[E_{j}^{k, n-1}+E_{j}^{k-1, n-1}\right] \Lambda_{0}^{2 \mid j, n-1}-\Gamma_{0}^{2 \mid k-1, n-2} \\
& +\sum_{j=0}^{2 \mid k, n-1}+\Gamma_{0}^{2 \mid k-1, n-1}-\Gamma_{0}^{2 \mid k-1, n-2} . \\
\Gamma_{0}^{k, n} \Lambda_{0}^{2 \mid j, n-1}-\sum_{j=1}^{k, n} E_{j}^{k, n} \Lambda_{0}^{2 \mid j-1, n-2} & \\
& +(n, q)+s(-n+1, q))\left[\sum_{j=1}^{k}(-1)^{j-1} E_{j}^{k, n} \Phi(n, j, 1)\right]
\end{aligned}
$$

A direct computation shows that $\Gamma_{0}^{2 \mid 2,5}=-s(2, q)$. Using the above relations between the $\Gamma_{0}^{2 \mid k, n}$, the Proposition now follows proving by induction the identities

$$
\Gamma_{0}^{2 \mid k, 2 k+1}=-s(k, q), \quad \Gamma_{0}^{2 \mid k, n}=-s(k, q)-s(n-k-1, q) .
$$

\section{The Exceptional Cases}

The most efficient way to verify the Reeder's Conjecture in the Exceptional cases is to compute explicitly the coefficients appearing in Stembridge's recurrences (evaluated in $-q$ and $q^{2}$ ) using a computer algebra software (in our case, SageMath 9.3 [20]) and prove that the polynomials computed by Gyoja, Nishiyama and Shimura for [12] satisfies such recurrences. We underline that a crucial ingredient for these computations is the strong efficiency of the Stembridge's algorithms. 
Table 2 Type $E_{6}$

Minuscule coweights: $\omega_{1}, \omega_{6}$

\section{Table 3 Type $E_{7}$}

Minuscule coweight: $\omega_{7}$

Table 4 Type $E_{8}$

Quasi-minuscule coweight: $\omega_{8}$

\section{Table 5 Type $F_{4}$}

Quasi-minuscule coweight: $\omega_{4}$

\section{Small representation}

Zero weight space

Highest weight $\phi_{\alpha, \beta}$ description

$\omega_{1}+\omega_{6}$

$\omega_{4}$

$\omega_{1}+\omega_{3}, \omega_{5}+\omega_{6}$

$3 \omega_{1}, 3 \omega_{6}$ $\phi_{20,2}$

$\phi_{30,3}+\phi_{15,5}$

$\phi_{64,4}$

$\phi_{24,6}$

\begin{tabular}{ll}
$\begin{array}{ll}\text { Small representation } \\
\text { Highest weight }\end{array}$ & $\begin{array}{l}\text { Zero weight space } \\
\phi_{\alpha, \beta} \text { description }\end{array}$ \\
\hline$\omega_{6}$ & $\phi_{27,2}$ \\
$\omega_{3}$ & $\phi_{56,3}+\phi_{21,6}$ \\
$2 \omega_{7}$ & $\phi_{21,3}$ \\
$\omega_{2}+\omega_{7}$ & $\phi_{120,4}+\phi_{105,5}$ \\
\hline
\end{tabular}

\begin{tabular}{ll}
\hline Small representation & $\begin{array}{l}\text { Zero weight space } \\
\phi_{\alpha, \beta} \text { description }\end{array}$ \\
\hline$\omega_{1}$ & $\phi_{35,2}$ \\
$\omega_{7}$ & $\phi_{112,3}+\phi_{28,8}$ \\
$\omega_{2}$ & $\phi_{210,4}+\phi_{160,7}$ \\
\hline
\end{tabular}

Small representation

Highest weight

$\omega_{3} \quad \phi_{8,3}^{\prime}+\phi_{1,12}^{\prime}$


As pointed out in [22], for cases $F_{4}$ and $E_{8}$ it is not possible to use minuscule recurrences (these algebrae do not have a minuscule coweight) and then we were forced to use Stembridge's quasi-minuscule recurrence ([22], Formula (5.13)), already used to prove Reeder's Conjecture in type $C$ (see [10]).

We list in Tables 2, 3, 4 and 5 the small weights different from $0, \theta_{s}$ and $\theta$ in the exceptional cases. We used the labeling of Dynkin diagrams as in [4]. The description of their zero weight spaces is exposed in $[1,19]$ using the notation of [6]. Moreover, for the $F_{4}$ case we used the computations contained in [3] and the results of Section 4.10 in [15].

We point out that, for type $E_{6}$, some nice symmetries can be used to reduce the number of cases that need to be examined. We remark that in Table 2 there are two pairs of weights that have the same zero weight space representation. Set $X_{1}=\left\{3 \omega_{1}, \omega_{1}+\omega_{3}\right\}$ and $X_{6}=\left\{3 \omega_{6}, \omega_{5}+\omega_{6}\right\}$. We recall that the automorphism of the $E_{6}$ Dynkin diagram that send labelled vertices 1 to 6 and 3 to 5 induces an involution $J$ on the weight lattice that pairwise exchange the weights in $X_{1}$ with weights in $X_{6}$. Let us denote by $R_{\lambda}^{\omega_{1}}$ (resp. $R_{\lambda}^{\omega_{6}}$ ) the minuscule recurrence for the weight $\lambda$, obtained choosing $\omega_{1}$ (resp. $\omega_{6}$ ) as a minuscule coweight. Fix $\lambda \in X_{1}$. We observed empirically that the involution $J$ sends $R_{\lambda}^{\omega_{1}}$ to $R_{J(\lambda)}^{\omega_{6}}$, preserving the coefficients appearing in the recurrences. This proves that $C_{\lambda}(q)$ and $C_{J(\lambda)}(q)$ satisfy the same recursive relations and then we reduced to prove Reeder's Conjecture only for weights in $X_{1}$. Our computations are available at the link in Bibliography [11].

Acknowledgments I am grateful to Professor Paolo Papi thank to whom I focused my attention on Reeder's Conjecture during my doctoral studies and who helped me with the computations for the exceptional cases. Moreover I would like to express my gratitude to Professor Kyo Nishiyama for sharing the computations of [12] for types $E$ and $F_{4}$. Finally I would like to thank Professor Martina Lanini, Professor Andrea Maffei, Francesco Ferrante and Alessandro Iraci for many useful discussions about computational issues and optimization strategies that $\mathrm{I}$ used in the $E_{8}$ and $F_{4}$ cases, and the anonymous referee for her/his helpful comments.

Data Availability Data sharing not applicable to this article.

Open Access This article is licensed under a Creative Commons Attribution 4.0 International License, which permits use, sharing, adaptation, distribution and reproduction in any medium or format, as long as you give appropriate credit to the original author(s) and the source, provide a link to the Creative Commons licence, and indicate if changes were made. The images or other third party material in this article are included in the article's Creative Commons licence, unless indicated otherwise in a credit line to the material. If material is not included in the article's Creative Commons licence and your intended use is not permitted by statutory regulation or exceeds the permitted use, you will need to obtain permission directly from the copyright holder. To view a copy of this licence, visit http://creativecommons.org/licenses/by/4.0/.

\section{References}

1. Achar, P.N., Henderson, A.: Geometric Satake, Springer correspondence and small representations. Sel. Math. New Ser. 19, 949-986 (2013)

2. Bazlov, Y.: Graded multiplicities in the exterior algebra. Adv. Math. 158, 129-153 (2001)

3. Beynon, W., Lusztig, G.: Some numerical results on the characters of exceptional Weyl groups. Math. Proc. Camb. Philos. Soc. 84(3), 417-426 (1978)

4. Bourbaki, N.: Lie Groups and Lie Algebras, Chapters 4-6. Springer, Berlin (2002)

5. Broer, A.: The sum of generalized exponents and Chevalley's restriction theorem for modules of covariants. Indag. Math. 6(4), 385-396 (1995)

6. Carter, R.W.: Finite Groups of Lie Type: Conjugacy Classes and Complex Characters. New York (1985)

7. De Concini, C., Möseneder Frajria, P., Papi, P., Procesi, C.: On special covariants in the exterior algebra of a simple Lie algebra. Rend. Lincei Mat. Appl. 25, 331-334 (2014) 
8. De Concini, C., Papi, P.: On some modules of covariants for a reflection group. Trans. Moscow Math. Soc., 257-273 (2017)

9. De Concini, C., Papi, P., Procesi, C.: The adjoint representation inside the exterior algebra of a simple Lie algebra. Adv. Math. 280, 21-46 (2015)

10. Di Trani, S.: On Reeder's Conjecture for Type $B$ and $C$ Lie algebras. Algebr. Represent. Theor. 25, 25-51 (2022)

11. Di Trani, S.: Computations for "Reeder's Conjecture for Even Orthogonal Lie algebras." https://sites. google.com/view/sabino-di-trani-web-page/home-page/publications

12. Gyoja, A., Nishiyama, K., Shimura, H.: Invariants for representations of Weyl groups and two-sided cells. J. Math. Soc. Japan 51, 1-34 (1999)

13. Kirillov, A., Pak, I.: Covariants of the symmetric group and its analogues in A. Weil algebras. (Russian) Funktsional. Anal. i Prilozhen. 24(3), 9-13, 96 (1990). translation in Funct. Anal. Appl. 24(3), 172-176 (1991)

14. Kostant, B.: Clifford algebra analogue of the Hopf-Koszul-Samelson theorem, the $\rho$-decomposition $c(\mathfrak{g})=E n d, v_{\rho} \otimes C(P)$, and the $\mathfrak{g}$-module structure of $\bigwedge \mathfrak{g}$. Adv. Math. 125, 275-350 (1997)

15. Lusztig, G.: Characters of Reductive Groups over a Finite Field. Princeton University Press, Princeton (1984)

16. Molchanov, V.: Poincarè series of representations of finite groups that are generated by reflections. (Russian) Funktsional. Anal. i Prilozhen. 26(2), 82-85 (1992). translation in Funct. Anal. Appl. 26(2), 143-145 (1992)

17. Reeder, M.: On the cohomology of compact Lie groups. L'Ensegnement Mathematique 41, 181-200 (1995)

18. Reeder, M.: Exterior Powers of the adjoint representation. Can. J. Math. 49, 133-159 (1997)

19. Reeder, M.: Zero weight spaces and the Springer correspondence. Indag. Math. 9, 431-441 (1998)

20. SageMath, the Sage Mathematics Software System (Version 8.1 and 9.3), The Sage Developers, 2017, https://www.sagemath.org

21. Stembridge, J.R.: First layer formulas for characters of $S L(n, \mathbb{C})$. Trans. Am. Math. Soc. 299(1), 319350 (1987)

22. Stembridge, J.R.: Graded multiplicities in the macdonald kernel. Part I. Int. Math. Res. Pap. 2005(4), $183-236(2005)$

Publisher's Note Springer Nature remains neutral with regard to jurisdictional claims in published maps and institutional affiliations. 